\title{
Oficina sobre sexualidade e namoro para pré-adolescentes ${ }^{1}$
}

\author{
Daniela Centenaro Levandowski ${ }^{2}$ \\ Universidade Federal de Ciências da Saúde de Porto Alegre, Porto Alegre-RS, Brasil \\ Márcia Moura Schmidt \\ Instituto de Cardiologia do Rio Grande do Sul, Porto Alegre-RS, Brasil
}

\begin{abstract}
Resumo: O presente trabalho tem por objetivo relatar as atividades realizadas em oficinas sobre sexualidade e namoro para pré-adolescentes, alunos de escolas públicas municipais e estaduais da cidade de Caxias do Sul-RS, no Programa Comunitário Cidadão do Século XXI. Tais oficinas visavam à troca de experiências e à reflexão sobre as ações e escolhas relativas ao exercício da sexualidade. Os encontros foram organizados de forma lúdica, por meio de dinâmicas de grupo ou atividades dirigidas, como leitura e discussão de textos e filmes, abordando temas diversos. Destaca-se a importância de ações de natureza preventiva para reduzir os riscos psicossociais, abrindo oportunidades para o desenvolvimento saudável dos adolescentes.
\end{abstract}

Palavras-chave: sexualidade, pré-adolescentes, prevenção, atividades.

\section{Workshop addressing sexuality and dating directed to adolescents}

\begin{abstract}
This paper reports activities carried out in workshops addressing sexuality and dating directed to adolescent students of public schools in Caxias do Sul/RS, Brazil included in the program Comunitário Cidadão do Século XXI [21 ${ }^{\text {st }}$ Century Citizen Community]. These workshops aimed to enable the exchange of experiences and reflection about sexuality-related actions and choices. The meetings were organized with a ludic approach, through group dynamics and directed activities such as reading and discussions on texts and movies, addressing several themes. The need to implement preventive actions to reduce psychosocial risk factors, giving these individuals opportunities to achieve healthy development is highlighted.
\end{abstract}

Keywords: sexuality, teenager, prevention, activities.

\section{Taller sobre sexualidad y relaciones entre enamorados para pre-adolescentes}

Resumen: La finalidad en este trabajo es relatar las actividades realizadas en talleres sobre sexualidad y relaciones entre enamorados para pre-adolescentes, alumnos de escuelas públicas municipales y estaduales de Caxias do Sul/RS, en el Programa Comunitario Cidadão do Século XXI. Tales seminarios tenían por objetivos el intercambio de experiencias y la reflexión sobre las propias acciones y preferencias relativas al ejercicio de la sexualidad. Los encuentros eran organizados de forma lúdica, por medio de dinámicas de grupo o actividades dirigidas, como lectura y discusiones de textos y películas, tratando de temas diversos. Se destaca la importancia de acciones de índole preventiva para reducir los riesgos psicosociales, abriendo oportunidades para el desarrollo sano de los jóvenes.

Palabras clave: sexualidad, pre-adolescentes, prevención, actividades.

No Brasil, o início da atividade sexual entre os adolescentes tem ocorrido cada vez mais precocemente. Esse início precoce acarreta muitas vezes a contaminação por doenças sexualmente transmissíveis (DSTs) ou a ocorrência de uma gestação não planejada (Borges, 2007).

Investigações realizadas em âmbito nacional e internacional tem indicado que a educação sexual promovida pela família e/ou pela escola contribui para postergar a iniciação

\footnotetext{
${ }^{1}$ Um recorte deste trabalho foi apresentado na X Jornada da Sociedade de Psicologia do Rio Grande do Sul, "Profissão: Psicólogo - Identidades, Práticas e Perspectivas", realizada em outubro de 2006. As autoras agradecem à Pró-Reitoria de Ação Comunitária da Universidade de Caxias do Sul (UCS), em especial à Coordenação e funcionários do Programa "Cidadão do Século XXI", bem como aos professores e alunos do Curso de Psicologia, que auxiliaram na concretização dessa proposta. Este texto foi revisado segundo Acordo Ortográfico da Língua Portuguesa (1990), em vigor a partir de $1^{\circ}$ de janeiro de 2009.

${ }^{2}$ Endereço para correspondência

Profa. Dra. Daniela Centenaro Levandowski. Universidade Federal de Ciências da Saúde de Porto Alegre. Departamento de Psicologia. Rua Sarmento Leite, 245 Sala 610. CEP 90050-170. Porto Alegre-RS, Brasil.. E-mail: danielal@ufcspa.edu.br
}

sexual dos adolescentes (Di Iorio, Kelley, \& HockenberryEaton, 1999; Romero, Medeiros, Vitalle, \& Wehba, 2007; Sousa, Fernandes, \& Barroso, 2006), para aumentar a taxa de utilização de métodos contraceptivos na primeira relação (Fisher, 1987; Mueller, Gavin, \& Kulkarni, 2008) e, dessa forma, para diminuir a possibilidade de ocorrência de uma gravidez indesejada e/ou de contaminação por uma DST (Camargo \& Botelho, 2007). Constata-se, assim, que a educação sexual parece ser efetiva na redução de comportamentos de risco entre os adolescentes (Blake, Simkin, Ledsky, Perkins, $\&$ Calabrese, 2001) especialmente quando acontece antes da sua iniciação (Buhi \& Goodson, 2007; Kohler, Manhart, \& Lafferty, 2008; Silva, 2002). Fica patente, então, a importância de atividades nesse âmbito para a prevenção de problemas de saúde reprodutiva e sexual entre os adolescentes.

De fato, a existência de programas dessa natureza nas escolas e em outras instituições comunitárias é imprescindível, uma vez que diversos estudos demonstram que mães, e principalmente os pais, abordam superficialmente o tema com os filhos, quando o fazem (Alves \& Brandão, 2009; Faustini, Novo, Cury, \& Juliano, 2003; Guimarães, Vieira, 
\& Palmeira, 2003; Romero e cols., 2007; Rosenthal \& Feldman, 1999; Sousa e cols., 2006). No cenário nacional, encontramos relatos de algumas intervenções junto a adolescentes no que toca à sexualidade, prevenção da gravidez e DSTs, implementadas em locais diversos (como escolas, unidades básicas de saúde ou universidades) por diferentes profissionais, algumas vezes com caráter interdisciplinar (Alencar, Silva, Silva, \& Diniz, 2008; Carvalho, Rodrigues, \& Medrado, 2005; Hassen, 2002; Jeolás \& Ferrari, 2003; Maheirie, Urnau, Vavassori, Orlandi, \& Baierle, 2005).

Infelizmente, existem poucos estudos avaliando a efetividade de programas de orientação sexual em nosso país. Além disso, resultados contraditórios tem sido encontrados a esse respeito em países como os Estados Unidos (DiCenso, Guyatt, Willan, \& Griffith, 2002; Kohler e cols., 2008; Silva, 2002). Contudo, tais contradições podem estar relacionadas à forma como os programas tem sido conduzidos naquele país, geralmente veiculando "mensagens de abstinência", que não proporcionam mudanças nos valores e atitudes sobre a relação sexual pré-marital (Sather \& Zinn, 2002). Além disso, restringir a informação sobre outras opções de cuidado com a saúde sexual e reprodutiva, em detrimento da abstinência, mostra-se uma questão eticamente controversa (Santelli e cols., 2006).

Desse modo, o oferecimento de um espaço de diálogo, pautado pelo acolhimento das dúvidas e o fornecimento de informações diretas e adequadas ao desenvolvimento e ao contexto do adolescente, parece ser a melhor forma para se abordar a temática da sexualidade. Nesse sentido, Vilella e Doreto (2006) defendem a produção de estratégias criativas para a abordagem do sexo seguro, que façam sentido para adolescentes de diferentes contextos socioculturais.

Fica evidenciada, dessa maneira, a necessidade de organizar oficinas criativas, envolvendo os participantes em um clima de confiança e abertura ao diálogo, sem preconceitos, com atividades diversificadas (tanto de cunho informativo, para abarcar o fornecimento de informações e o esclarecimento de dúvidas, quanto lúdico e vivencial, para estimular a reflexão sobre essas temáticas).

Diante disso, o presente trabalho teve por objetivo relatar as atividades realizadas em oficinas sobre sexualidade e namoro para pré-adolescentes, alunos de escolas públicas municipais e estaduais da cidade de Caxias do Sul-RS, realizadas por alunos do Curso de Psicologia da Universidade de Caxias do Sul (UCS), sob supervisão das autoras, junto ao Programa Comunitário Cidadão do Século XXI, entre os anos de 2004 e 2005.

\section{Método}

O referido Programa estava vinculado à Pró-Reitoria de Ação Comunitária da UCS, tendo sido criado no ano 2000 para o atendimento de crianças e adolescentes no turno oposto ao da escola, com o objetivo de cumprir com o papel social da universidade, através da promoção de melhores condições de vida para a população, a partir da educação em colaboração com as famílias, escolas e organizações comunitárias.

Para tanto, eram oferecidas aos participantes, todos os dias da semana, pela manhã e pela tarde, atividades organizadas na forma de oficinas, contemplando temas variados, tais como: primeiros socorros, língua estrangeira, direitos e cidadania, educação ambiental, literatura, teatro, trabalhos manuais, esportes, informática. Tais oficinas eram ministradas voluntariamente por alunos de diferentes cursos de graduação da universidade, sob supervisão de professores, assim como por pessoas da comunidade.

No ano de 2004, foram atendidos pelo Programa quatro mil alunos de quinta à oitava série, tendo havido uma pequena redução neste número no ano de 2005. Ao ingressarem, crianças e adolescentes escolhiam as oficinas das quais desejavam participar, sendo obrigatória apenas a matrícula na oficina Preparação para a Vida (PPV), que abordava temas diversos (educação no trânsito, primeiros socorros, etiqueta e boas maneiras, autoestima, DSTs, higiene, dentre outros).

Quando as autoras passaram a integrar a equipe do Programa, verificaram que a inserção da Psicologia estava acontecendo através de atividades de educação sexual na oficina PPV. Apesar disso, observava-se o expressivo interesse pelo assunto "sexualidade", já que o mesmo era frequentemente citado, nas avaliações dessa oficina, como um tema a ser abordado em outras oportunidades. Assim, ficava evidente a necessidade de oferecer uma atividade que tratasse especificamente dos temas namoro e sexualidade, contemplando não apenas a transmissão de informações, mas, principalmente, as questões emocionais ligadas a essas temáticas. O alto número de participantes nas oficinas dificultava a busca por informações e uma troca mais genuína entre eles, indicando a necessidade de se trabalhar com grupos menores.

Dessa forma, organizou-se uma oficina, denominada Namoro e Sexualidade. Sua implantação tinha por intuito proporcionar um espaço para a troca de experiências e para a discussão de assuntos relativos aos relacionamentos e à sexualidade em geral, oportunizar a reflexão sobre as consequências das próprias ações e as escolhas relativas ao exercício da sexualidade e informar sobre métodos contraceptivos e DSTs.

\section{Participantes}

Participaram das oficinas Namoro e Sexualidade aproximadamente 270 alunos de quinta à oitava série do Ensino Fundamental de escolas públicas municipais e estaduais da cidade de Caxias do Sul, RS, de 12 a 15 anos de idade, matriculados no Programa Cidadão do Século XXI.

\section{Considerações éticas}

As oficinas Namoro e Sexualidade foram executadas a partir dos princípios éticos de autonomia, ou seja, capacidade de pensar e agir com conhecimento e sem coação externa; de beneficência e de não maleficência, oferecendo o máximo de benefício com o mínimo de dano e de justiça, garantindo 
que cada pessoa fosse tratada como é moralmente correto e apropriado, com justa distribuição dos recursos disponíveis (Gobbetti \& Cohen, 2005).

A proposta das oficinas era avaliada pela Coordenação do Programa e outros docentes. Além disso, os participantes optaram voluntariamente pela matrícula na atividade e tiveram a liberdade de interromper a sua participação a qualquer momento, simplesmente pelo seu não comparecimento aos encontros.

Outro cuidado tomado já no primeiro encontro foi o de fornecer uma explicação acerca dos objetivos da oficina, para que os adolescentes pudessem avaliar a pertinência de sua participação, e elaborar um contrato, visando a preservar a confidencialidade e promover um clima de respeito e confiança diante dos assuntos abordados.

\section{Procedimentos}

Nesta seção serão descritos os aspectos técnicos que nortearam a realização das oficinas. O trabalho foi realizado ao longo de três semestres (2004/I e II, 2005/I), tendo sido oferecidos seis horários por semestre (seis turmas). Cada oficina era desenvolvida em 12 encontros semanais, de 1h30min de duração. Em média cada turma tinha 15 participantes, sendo aproximadamente $70 \%$ do sexo feminino.

As atividades eram planejadas pelas autoras, em conjunto com alunos de diferentes semestres do Curso de Psicologia da UCS. Estes trabalhavam em duplas, quando possível, responsabilizando-se pela execução das atividades. Cada autora supervisionava um grupo de alunos, semanalmente, visando ao acompanhamento das atividades e a sua adequação às necessidades de cada grupo. Nesses momentos, os alunos também discutiam as suas experiências e dificuldades na condução das oficinas.

Todos os encontros foram organizados de forma lúdica, por meio de dinâmicas de grupo ou atividades dirigidas, como leitura e discussão de textos e filmes. Entretanto, nenhuma das oficinas realizadas ao longo dos três semestres foi exatamente igual à outra, uma vez que os assuntos e as técnicas utilizadas foram adaptados às características de cada grupo.

Ainda assim, como algumas atividades foram compartilhadas, pode-se dizer que, em linhas gerais, os encontros ocorreram da seguinte forma: no primeiro dia era realizada uma técnica de apresentação e integração dos participantes. Também se fazia um rapport sobre o funcionamento dos encontros, um contrato de sigilo e o levantamento dos assuntos de interesse do grupo, por escrito e sem identificação. No segundo encontro, por exemplo, se propunha que, em duplas, preenchessem uma ficha com as características das meninas e dos meninos em três momentos distintos do desenvolvimento: aos cinco, aos dez e aos 15 anos. Nas apresentações eram debatidas as diferenças de gênero e de papéis sociais, propondo-se inclusive outra dinâmica de grupo, objetivando a troca de papéis sexuais: os meninos deveriam argumentar as razões pelas quais era melhor ser menina e vice-versa.
Em outro encontro, os participantes eram convidados a completar uma tabela, em pequenos grupos, com informações sobre a situação atual dos adolescentes. Tal tabela abordava as principais mudanças históricas e sociais da adolescência desde os anos 60. Após isso, havia uma discussão sobre essa temática, valorizando-se as percepções dos participantes sobre o assunto.

O filme Aos Treze foi projetado em algumas oficinas, por sugestão dos próprios participantes, tendo sido realizada após uma discussão sobre o mesmo, com esclarecimento de dúvidas sobre a adolescência. A partir do quinto encontro, os temas levantados pelos participantes no primeiro dia passaram a ser enfatizados: puberdade, primeiro beijo, ficar, sedução, namoro e relação sexual. Montagens utilizando artigos de revistas teens foram feitas para suscitar os debates, assim como, em alguns grupos, foram usadas técnicas como baralho com perguntas e dramatizações.

Quanto à puberdade, foi distribuído um pequeno texto que indicava as mudanças corporais dos meninos e das meninas, comparando-as. Após a leitura desse texto, faziase uma discussão das suas principais ideias, bem como um levantamento de dúvidas sobre o tema, com o posterior esclarecimento das mesmas. Para abordar os temas "ficar e namoro", utilizou-se a música Já sei namorar, dos Tribalistas, bem como uma crônica de Arnaldo Jabor que enfatizava a efemeridade dos relacionamentos no cenário atual. Em outro encontro os alunos foram solicitados a confeccionar, em pequenos grupos, um painel sobre o tema "Paquerar", que podia conter exemplos de situações e/ou o que eles entendiam sobre o assunto, assim como suas dúvidas. Após a confecção, que em geral se estendia por mais de um encontro, havia um momento para apresentação dos cartazes e discussão com o grande grupo, visando ao esclarecimento de dúvidas.

Já o tema da relação sexual foi discutido a partir de um material lúdico, em formato de histórias em quadrinhos, contendo cenas e diálogos de jovens e seus familiares apontando razões para adiar ou não a primeira relação sexual. Cada participante assinalava as alternativas que julgava mais apropriadas e depois era feita uma discussão em grande grupo, analisando-se as razões para se ter e não se ter relação sexual. Os alunos da psicologia enfatizavam a discussão das ansiedades, expectativas e medos (do desconhecimento, de não impressionar, do futuro), bem como dos sentimentos de vergonha e culpa, dentre outros, conforme o ritmo e a curiosidade de cada grupo.

Ainda com o objetivo de esclarecer as dúvidas e de atender aos interesses levantados pelos participantes no primeiro encontro, as principais DSTs foram apresentadas pelos alunos da psicologia de forma expositiva e posteriormente debatidas, assim como os métodos contraceptivos. Foi realizado inclusive um treinamento para o uso da camisinha com um pênis de borracha. Nesses encontros, alguns grupos foram divididos conforme o sexo dos participantes, a fim de que as dúvidas pudessem ser sanadas sem maiores constrangimentos. 
Ao término da oficina, foram realizadas avaliações com os adolescentes, por escrito e sem identificação, para se obter um feedback da atividade. A avaliação das atividades foi realizada por meio de questões bem simples, abertas, solicitando aos participantes que mencionassem pontos positivos e negativos da oficina, assim como o que eles consideravam que poderia ser diferente em uma próxima oportunidade. As respostas foram lidas e classificadas pelas duas autoras, sendo utilizadas para o planejamento das atividades do semestre seguinte. Uma avaliação também era realizada pelos alunos do Curso de Psicologia que ministravam as oficinas.

\section{Análise dos dados}

Foi realizada uma análise qualitativa e quantitativa das respostas das avaliações dos adolescentes, bem como usados os registros do diário de campo do grupo e as reflexões das coordenadoras das oficinas.

\section{Resultados e Discussão}

A primeira atividade realizada após a integração dos participantes salientava os diferentes estágios do desenvolvimento, visando à reflexão sobre o aumento da autonomia e o consequente aumento da responsabilidade ao longo da vida. Esperava-se estimular uma conscientização sobre as decisões que os jovens devem tomar frente à sua vida sexual $\mathrm{e}$ reprodutiva. A referida atividade visava também estimular as discussões sobre a identidade de gênero e o desenvolvimento dos papéis sociais. Nesse sentido, nos debates realizados, os participantes com frequência concluíam que ser menino era melhor que ser menina, pois não menstruavam, podiam sair à noite e eram menos cobrados pelos familiares. Tais achados revelam as concepções sociais e culturais tradicionais acerca dos papéis de gênero (Traverso-Yépez \& Pinheiro, 2005).

Em função disso, propunha-se a dinâmica da troca de papéis, na qual os meninos deveriam argumentar as razões pelas quais era melhor ser menina e vice-versa. Cabia aos alunos de psicologia discutir acerca das ansiedades e angústias diante dessa "troca". Por exemplo, o quanto para os meninos era difícil desempenhar o papel de "sabe tudo" e o quanto era complicado demonstrar afetos, diante do estigma social de que "meninos não choram". Em relação às meninas, destacou-se a preocupação em relação à imagem, ao julgamento alheio, ao medo da entrega sexual e do arrependimento posterior. Novamente foram reveladas algumas concepções sociais e culturais tradicionais acerca da feminilidade e da masculinidade (Altmann, 2007; Borges, 2007; Santos \& Silva, 2008).

Em alguns grupos, durante a execução dessa atividade, foi possível identificar meninos com um comportamento hostil e agressivo, o que fez emergir uma discussão sobre a violência como forma de autoafirmação diante das exigências de "ser macho". Tal ideia também foi veiculada pelos adolescentes participantes do estudo de Traverso-Yépez e Pinheiro (2005). As discussões sobre esse tema também geraram reflexões sobre as relações familiares e a imitação de modelos de relacionamento aprendidos nesse âmbito, uma vez que tal aprendizagem, conforme as autoras, facilita a perpetuação da violência de gênero, estendendo-se às demais relações dos adolescentes.

A atividade acerca das mudanças históricas e sociais da adolescência desde os anos 60 até os anos 2000 visava ressaltar a liberdade e a autonomia atingidas nos dias atuais pelos adolescentes, especialmente em relação à sexualidade, e as modificações das relações familiares e amorosas, refletindo sobre as consequências dessas mudanças. Esse objetivo foi plenamente atingido, pois todos os grupos chegavam à conclusão de que, apesar das dificuldades sentidas, tais como as pressões dos amigos e da família e as restrições sociais, era mais fácil ser adolescente nos dias atuais do que em épocas anteriores, visto que tinham mais liberdade para expressar ideias e sentimentos e também para fazer escolhas de vida.

Em relação ao filme Aos Treze, as discussões centraram-se nas influências do grupo de amigos, na importância do sentimento de pertença e da popularidade e nas consequências desses aspectos para os adolescentes. Nos debates, os alunos da Psicologia eram orientados a questionar os participantes sobre até que ponto deve-se modificar o próprio comportamento para ser aceito pelo grupo de amigos, a fim de ressaltar o respeito às diferenças. Este aspecto reveste-se de importância, pois o grupo de iguais foi identificado por Taquete e Vilhena (2008), entre adolescentes do sexo feminino, como um estímulo para a atividade sexual, principalmente para aquelas jovens que julgavam que esses iguais já haviam tido sua primeira experiência sexual.

Cabe salientar que, no levantamento das dúvidas e dos assuntos de interesse dos participantes, realizado sempre no primeiro encontro, em geral identificava-se temas semelhantes a serem desenvolvidos (ficar, beijar, transar e namorar, permeados por conflitos em relação ao desejo versus as normas e valores morais e as noções de certo e errado, a opinião da família versus a opinião dos amigos). Outros pontos de preocupação, interesse e dúvida eram as DSTs e os métodos contraceptivos. Tal panorama assemelha-se ao encontrado em outros estudos de intervenção realizados com adolescentes sobre essa temática (Alencar e cols., 2008; Carvalho e cols., 2005; Hassen, 2002; Jeolás \& Ferrari, 2003; Maheirie e cols., 2005).

As ansiedades foram expressas principalmente quanto ao primeiro beijo e ao ficar, em detrimento da primeira relação sexual. Tal achado pode ser explicado em parte pela idade dos participantes, que se defrontavam, naquele momento, com essas vivências. As meninas, talvez por demonstrarem mais suas inseguranças, queriam saber como se beijava e se havia técnicas específicas para a aprendizagem do beijo, assim como técnicas de paquera. Tal achado permitiu identificar a necessidade das meninas de apresentarem um bom desempenho para agradarem os meninos, dados também encontrados no estudo de Santos e Silva (2008), a partir da análise de revistas teens. Conforme esses autores, tais dados 
refletem uma assimetria afetiva e sexual entre homens e mulheres no que tange à sexualidade e ao amor. Contudo, após a realização das atividades da oficina, os participantes concluíam que cada um tem seu estilo de beijar e de paquerar, relacionado ao seu jeito de ser.

Em relação aos métodos contraceptivos, treinava-se a colocação da camisinha e discutiam-se mitos como usar camisinha é como chupar bala com papel (Toneli \& Vavassori, 2004). A importância do uso desta para a prevenção de uma gestação não programada e das DSTs, não só do HIV/AIDS, também foi muito salientada e debatida, principalmente em relação ao Papiloma Vírus Humano (HPV), causa de câncer de útero e colo. Aqui, dois aspectos mereceram destaque: primeiro, muito embora soubessem da importância da camisinha, a maioria desconhecia sua correta colocação. Em segundo lugar, a informação sobre a camisinha como meio de proteção, até mesmo para doenças como o câncer, impressionou particularmente as meninas, que desconheciam esse tipo de risco. Infelizmente, não foi feita uma avaliação sistemática para verificar diferenças no nível de conhecimento dos participantes sobre essas temáticas antes e depois da participação na oficina, como constou no estudo de Faustini e cols. (2003), no qual foi identificado um aumento nos conhecimentos de adolescentes que frequentaram encontros informativos realizados por uma equipe multiprofissional.

Por fim, em geral as oficinas foram avaliadas positivamente pelos participantes. Estes, apesar de certo constrangimento e nervosismo inicial, ao longo dos encontros, a partir da percepção da proposta da oficina, sentiam-se mais à vontade para participar, expondo suas dúvidas e opiniões. A grande maioria revelou ter finalizado a atividade com um nível maior de esclarecimento e conscientização sobre os temas trabalhados, já que geralmente não possuía um espaço para o diálogo no ambiente doméstico, dado esse que concorda com a literatura revisada (Alves \& Brandão, 2009; Guimarães e cols., 2003; Romero e cols., 2007; Rosenthal \& Feldman, 1999; Sousa e cols., 2006). A título de ilustração, na avaliação realizada em 2004/II, 29,4\% dos participantes destacaram a aprendizagem como ponto positivo dos encontros e 17,6\% ressaltaram a importância de esclarecer as dúvidas acerca de assuntos sobre os quais não costumavam falar com seus pais.

$\mathrm{Na}$ última avaliação realizada, já no ano de 2005 $(2005 / \mathrm{I})$, pode-se observar que a atividade que os participantes mais gostaram foi aquela informativa sobre o uso da "camisinha e sobre as DSTs" (44,44\%), seguida pelo filme Aos Treze (22,22\%) e por "todas as atividades" (22,22\%). Quanto às atividades que menos gostaram, 66,66\% dos respondentes disseram que "de nenhuma", o que demonstrou uma boa aceitação do trabalho realizado. Em relação ao que poderia ser diferente, 33,33\% responderam "nada" e 22,22\% solicitaram mais horas de "aula" referindo-se ao desejo de continuação dos encontros. Em função desses dados, acredita-se que a oficina tenha cumprido seus principais objetivos.

\section{Considerações finais}

A sexualidade é um elemento central na formação da identidade do adolescente, envolvendo a imagem corporal, a descoberta do outro como objeto de amor ou de desejo, a descoberta de si mesmo e das diversas relações com familiares, grupos e profissionais (Romero e cols., 2007). De fato, sabe-se da importância desse tema e da mobilização dos adolescentes frente a essas vivências, que muitas vezes não são compartilhadas com familiares e amigos, por medo de críticas e repreensões. Tal realidade abre espaços de intervenção valiosos para os profissionais da saúde, incluindo-se aí a Psicologia.

Assim, o desenvolvimento de um trabalho de educação sexual com adolescentes não atua apenas na minimização de um problema de saúde pública, como é o caso da contaminação por DSTs/HIV ou de uma gestação não planejada, mas também na promoção da saúde integral desses jovens. Contudo, para atingir esse fim, um programa de educação sexual para esse público deve ser oferecido de maneira divertida e criativa, com o objetivo de encorajar discussões e desenvolver um entendimento compartilhado das temáticas. Deve funcionar questionando mitos e preconceitos e orientando para o autoconhecimento e para o bem-estar biopsicossocial (Carvalho e cols., 2005; Jeolás \& Ferrari, 2003), como se objetivou no presente trabalho.

Carvalho, Morais, Koller e Piccinini (2007) referem que os profissionais da psicologia deveriam participar de atividades de promoção de fatores de proteção e da resiliência nos mais diferentes contextos, a fim de trabalhar na direção da saúde integral das pessoas. Considera-se que as ações da psicologia nas oficinas Namoro e Sexualidade tiveram esse caráter preventivo e promotor de saúde física e mental, pois proporcionaram espaços para reflexões, orientações e esclarecimentos de dúvidas, assim como estimularam aspectos sadios da personalidade dos participantes, fomentando especialmente o desenvolvimento das capacidades de discussão, antecipação, planejamento e autocuidado.

Nessa perspectiva, tais oficinas também contribuíram para qualificar a formação de diversos alunos do curso de psicologia, propiciando a inserção em atividades de cunho preventivo, com foco na promoção da saúde e nos aspectos sadios do desenvolvimento humano, que, segundo Calvetti, Muller e Nunes (2007), deveriam ser cada vez mais contempladas na formação de novos profissionais psicólogos.

Especificamente em relação às atividades desenvolvidas, de modo geral mostraram-se satisfatórias para o alcance dos objetivos propostos. Nesse sentido, o presente relato pretende ser um estímulo para que outros profissionais divulguem suas experiências de trabalho, contribuindo para a elaboração de atividades inovadoras, adequadas às necessidades de saúde e bem-estar de crianças e adolescentes. Sugestões para novos estudos envolvem a inclusão de pais e professores nas atividades de educação sexual, uma vez que 
família e escola são importantes contextos de desenvolvimento humano (Dessen \& Polônia, 2007) e devem atuar de modo integrado em relação a esse aspecto formativo, bem como a realização de uma avaliação sistemática da efetividade de tais programas.

\section{Referências}

Alencar, R. A., Silva, L., Silva, F. A., \& Diniz, R. E. S. (2008). Desenvolvimento de uma proposta de educação sexual para adolescentes. Ciência \& Educação, 14, 159168.

Alves, C. A., \& Brandão, E. R. (2009). Vulnerabilidades no uso de métodos contraceptivos entre adolescentes e jovens: Interseções entre políticas públicas e atenção à saúde. Ciência \& Saúde Coletiva, 14, 661-670.

Altmann, H. (2007). Educação sexual e primeira relação sexual: Entre expectativas e prescrições. Estudos Feministas, 15, 333-356.

Blake, S. M., Simkin, L., Ledsky, R., Perkins, C., \& Calabrese, J. M. (2001). Effects of a parent-child communications intervention on young adolescents' risk for early onset of sexual intercourse [Versão eletrônica]. Family Planning Perspectives, 33, 52-61. Recuperado em 20 fevereiro 2009, de http://www.guttmacher.org/ pubs/journals/3305201.html

Borges, A. L. V. (2007). Relações de gênero e iniciação sexual de mulheres adolescentes. Revista da Escola de Enfermagem da USP, 41, 597-604.

Buhi, E. R., \& Goodson, P. (2007). Predictory of adolescent sexual behavior and intention: A theory guided systematic review. Journal of Adolescent Health, 40, 4-21.

Calvetti, P. Ü., Muller, M. C., \& Nunes, M. L. T. (2007). Psicologia da Saúde e Psicologia Positiva: Perspectivas e desafios. Psicologia: Ciência e Profissão, 27, 706-717.

Camargo, B. V., \& Botelho, L. J. (2007). AIDS, sexualidade e atitudes de adolescentes sobre proteção contra o HIV. Revista de Saúde Pública, 41, 61-68.

Carvalho, A. M., Rodrigues, C. S., \& Medrado, K. S. (2005). Oficinas em sexualidade humana com adolescentes. Estudos de Psicologia (Natal), 10, 377-384.

Carvalho, F. T. de, Morais, N. A. de, Koller, S. H., \& Piccinini, C. A. (2007). Fatores de proteção relacionados à promoção de resiliência em pessoas que vivem com HIV/AIDS. Cadernos de Saúde Pública, 23, 2023-2033.

Dessen, M. A., \& Polônia, A. da (2007). A família e a escola como contextos de desenvolvimento humano. Paidéia (Ribeirão Preto), 17, 21-32.

Di Iorio, C., Kelley, M., \& Hockenberry-Eaton, M. (1999). Communication about sexual issues: Mothers, fathers and friends. Journal of Adolescent Health, 24, 181-189.

DiCenso, A., Guyatt, G., Willan, A., \& Griffith, L. (2002). Interventions to reduce unintended pregnancies among adolescents: Systematic review of randomised controlled trials. British Medical Journal, 324, 1426.
Faustini, D. M. T., Novo, N. F., Cury, M. C. F. S., \& Juliano, Y. (2003). Programa de orientação desenvolvido com adolescentes em centro de saúde: Conhecimentos adquiridos sobre os temas abordados por uma equipe multidisciplinar. Ciência \& Saúde Coletiva, 8, 783-790.

Fisher, T. D. (1987). Family communication and the sexual behavior and attitudes of college students. Journal of Youth and Adolescence, 16, 481-495.

Guimarães, A. M. A. N., Vieira, M. J., \& Palmeira, J. A. (2003). Informações dos adolescentes sobre métodos anticoncepcionais. Revista Latino-Americana de Enfermagem, 11, 293-298.

Gobbetti, G. J., \& Cohen, C. (2005). Bioética: Desenvolvimento humano e os profissionais de saúde. In S. M. C. Ismael (Org.), Temas de prevenção, ensino e pesquisa que permeiam o contexto hospitalar (pp. 149162). São Paulo: Casa do Psicólogo.

Hassen, M. N. A. (2002). Grupos focais de intervenção no projeto Sexualidade e Reprodução. Horizontes Antropológicos, 8(17), 159-177.

Jeolás, L. S., \& Ferrari, R. A. P. (2003). Oficinas de prevenção em um serviço de saúde para adolescentes: Espaço de reflexão e de conhecimento compartilhado. Ciência \& Saúde Coletiva, 8, 611-620.

Daniela Centenaro Levandowski é Professora Adjunta I do Departamento de Psicologia da Universidade Federal de Ciências da Saúde de Porto Alegre (UFCSPA), bolsista de Produtividade em Pesquisa do CNPq.

Márcia Moura Schmidt é doutoranda em Ciências da Saúde: Cardiologia pelo Programa de Pós-graduação do Instituto de Cardiologia do Rio Grande do Sul, Fundação Universitária de Cardiologia, IC-FUC, integrante do Grupo de Pesquisa: Hemodinâmica e psicóloga do Instituto de Cardiologia do Hospital de Viamão, RS.
Recebido: 20/05/2008

$1^{a}$ revisão: 03/03/2009

$2^{a}$ revisão: $18 / 04 / 2009$

$3^{a}$ revisão: $16 / 06 / 2009$

Aceite final: 23/07/2009 